\title{
Search for top squarks and dark matter particles in opposite-charge dilepton final states at CMS
}

\section{Daniel Spitzbart ${ }^{* \dagger}$}

Austrian Academy of Sciences

E-mail: daniel.spitzbartecern.ch

\begin{abstract}
A search for new physics is presented in final states with two oppositely charged leptons, jets identified as originating from b quarks, and missing transverse momentum using 35.9/fb of CMS data recorded in 2016. Hypothetical signal events are efficiently separated from the dominant top quark pair background with requirements on missing transverse momentum and transverse mass variables, the latter reducing the background by four orders of magnitude. No significant deviation is observed from the expected background. The results are interpreted in terms of simplified models of pair produced scalar partners of top quarks (top squarks), as predicted by supersymmetric models. Exclusion limits reach up to top squark masses of $1.3 \mathrm{TeV}$ for specific model assumptions. Additionally, pair production of dark matter particles via scalar or pseudoscalar mediators is tested, and the analysis provides exclusions of scalar mediators with masses below $100 \mathrm{GeV}$.
\end{abstract}

${ }^{\dagger}$ on behalf of the CMS Collaboration. 


\section{Introduction}

The standard model of particle physics (SM) successfully describes processes at very different energy scales, reaching up to the $\mathrm{TeV}$ scale which is probed at the Large Hadron Collider (LHC) at CERN. However, several shortcomings are present within the SM that give hints that physics beyond this theory exists. Supersymmetry (SUSY) is one of the most prominent theories that provides a solution to the hierarchy problem, and, if R-parity is conserved, a dark matter candidate in form of the lightest SUSY particle. The lightest SUSY particles are expected to have masses at the TeV scale, accessible with the LHC.

A search for top squark pair production in final states with two opposite-charge leptons, at least two hadronic jets with one of them being identified to originate from $b$ quarks (b jet), and significant transverse momentum imbalance $\left(p_{\mathrm{T}}^{\text {miss }}\right)$, is performed [1]. The analysis is based on a dataset of proton-proton collisions at a center-of-mass energy of $13 \mathrm{TeV}$, recorded by the CMS experiment in 2016 [2]. Diagrams for processes leading to such final states are shown in Fig. 1. In the simplest SUSY model, shown in Fig. 1 (left), the pair produced top squarks each deacy to the stable lightest neutralino $\widetilde{\chi}_{1}^{0}$ (LSP) and a top quark. Following the conventions of [3], this model is

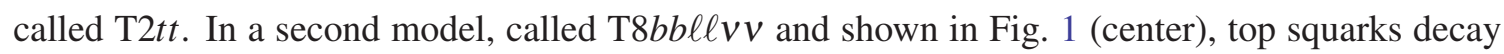
via intermediate charginos to sleptons, which subsequently decay to a lepton and a neutralino $\widetilde{\chi}_{1}^{0}$. This decay is motivated by the $100 \%$ branching fraction into two leptons. In a simplified dark matter (DM) model shown in Fig. 1 (right) a scalar $(\phi)$ or pseudoscalar $(a)$ mediator is produced in the $t \bar{t} \mathrm{t}$-channel that decays to two Dirac fermions $\chi$, which are the stable DM particles.
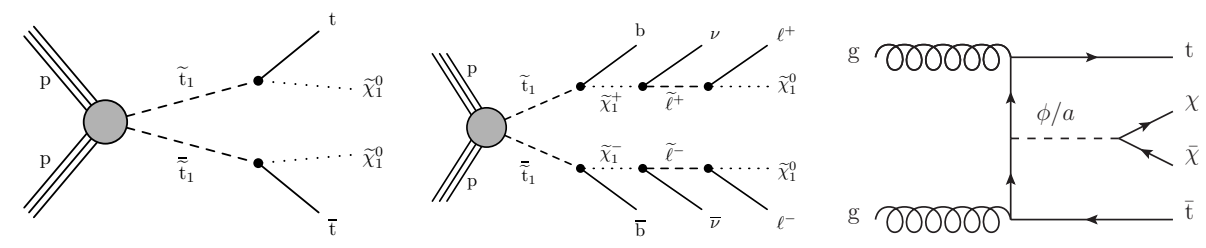

Figure 1: Diagrams for simplified SUSY and dark matter models: strong production of top squark pairs $\widetilde{\mathrm{t}}_{1} \overline{\mathrm{t}}_{1}$, where each top squark decays to a top quark and a $\widetilde{\chi}_{1}^{0}$ (T2tt model, left), or to a neutrino and an intermediate

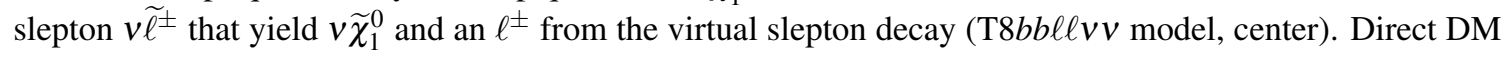
production through scalar or pseudoscalar mediators in association with top quarks is shown at the right.

\section{Search strategy}

We select events with two leptons, electrons or muons, with a transverse momentum $\left(p_{\mathrm{T}}\right)$ of at least $20 \mathrm{GeV}$ and no additional leptons with $p_{\mathrm{T}}>15 \mathrm{GeV}$. At least two jets, out of which at least one has to be classified as b jet, are required. In order to suppress contributions from Drell-Yan (DY) production we require the invariant mass of same-flavor lepton pairs to be more than $15 \mathrm{GeV}$ off the $\mathrm{Z}$ boson mass. By requiring $p_{\mathrm{T}}^{\text {miss }}>80 \mathrm{GeV}$ and a minimum value for a measure of the significance of $p_{\mathrm{T}}^{\text {miss }}, \mathrm{DY}$ and multiboson processes are reduced further. Requirements on the angular separation of $p_{\mathrm{T}}^{\text {miss }}$ with the leading and subleading jets suppress events that pass the aforementioned selection due to jet mismeasurements and the $p_{\mathrm{T}}^{\text {miss }}$ resolution. 


\begin{tabular}{|c|c|c|c|c|}
\hline$M_{\mathrm{T} 2}(b \ell b \ell)(\mathrm{GeV})$ & $p_{\mathrm{T}}^{\mathrm{miss}}(\mathrm{GeV})$ & $100 \leq M_{\mathrm{T} 2}(\ell \ell)<140 \mathrm{GeV}$ & $140 \leq M_{\mathrm{T} 2}(\ell \ell)<240 \mathrm{GeV}$ & $M_{\mathrm{T} 2}(\ell \ell) \geq 240 \mathrm{GeV}$ \\
\hline \hline \multirow{2}{*}{$0-100$} & $80-200$ & SR0 & SR6 & \\
\cline { 2 - 4 } & $\geq 200$ & SR1 & SR7 & \multirow{2}{*}{ SR12 } \\
\hline \multirow{2}{*}{$100-200$} & $80-200$ & SR2 & SR8 & \\
\cline { 2 - 4 } & $\geq 200$ & SR3 & SR10 & \\
\hline \multirow{2}{*}{$\geq 200$} & $80-200$ & SR4 & SR11 & \\
\cline { 2 - 4 } & $\geq 200$ & SR5 & SR & \\
\hline
\end{tabular}

Table 1: Definition of the signal regions, which are further split into opposite-flavor and same-flavor regions.

After applying the above selection criteria the majority of background events contain two leptons from top quark pair production (țt), where both the top quark and antiquark decay leptonically. A variable called $M_{\mathrm{T} 2}(\ell \ell)$ based on the transverse mass $\left(M_{\mathrm{T}}\right)$ is constructed in order to separate potential signal events from the dominant background,

$$
M_{\mathrm{T} 2}(\ell \ell)=\min _{\vec{p}_{\mathrm{T}, 1}^{\text {miss }}+\vec{p}_{\mathrm{T}, 2}^{\text {miss }}=\vec{p}_{\mathrm{T}}^{\text {miss }}}\left(\max \left[M_{\mathrm{T}}\left(\vec{p}_{\mathrm{T}, 1}^{\mathrm{vis}}, \vec{p}_{\mathrm{T}, 1}^{\text {miss }}\right), M_{\mathrm{T}}\left(\vec{p}_{\mathrm{T}, 2}^{\mathrm{vis}}, \vec{p}_{\mathrm{T}, 2}^{\text {miss }}\right)\right]\right) .
$$

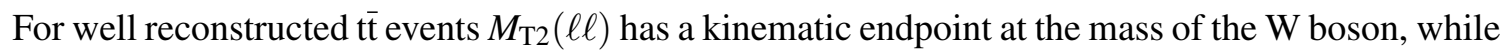
signal events would also populate the tails of the distributions due to the additional contribution of the LSPs to $p_{\mathrm{T}}^{\text {miss }}$. One can furthermore add the momentum of the $\mathrm{b}$ jets to the visible momentum component in the computation of $M_{\mathrm{T} 2}(\ell \ell)$, resulting in a variable called $M_{\mathrm{T} 2}(b \ell b \ell)$ with a kinematic endpoint at the top quark mass for $\bar{t} \bar{t}$ events.
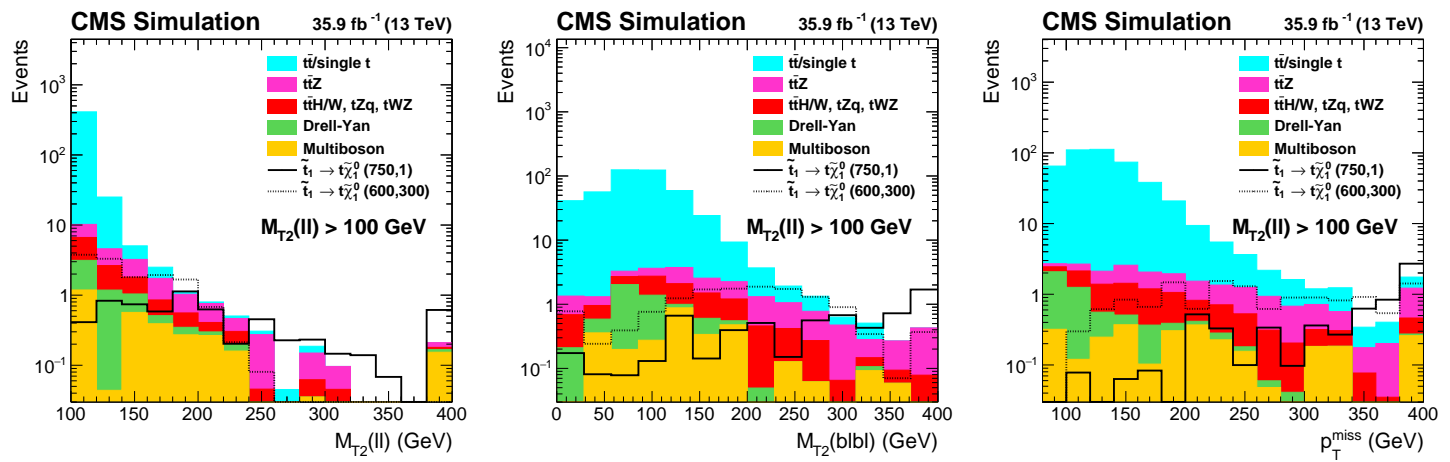

Figure 2: Simulated distributions of $M_{\mathrm{T} 2}(\ell \ell)$ (left), $M_{\mathrm{T} 2}(b \ell b \ell)$ (center), and $p_{\mathrm{T}}^{\text {miss }}$ (right) for the SM processes (stacked filled histograms) and a SUSY signal with a top squark mass of 750 (600) GeV and the LSP mass of 1 (300) $\mathrm{GeV}$ shown as solid (dashed) black line [1].

Together with $p_{\mathrm{T}}^{\text {miss }}, M_{\mathrm{T} 2}(\ell \ell)$ and $M_{\mathrm{T} 2}(b \ell b \ell)$ are the main search variables in this analysis. Several signal regions are constructed using various thresholds of those observables which are introduced in Table 1. Simulated distributions of the variables are shown in Fig. 2.

\section{Background prediction}

The main contributions to backgrounds from SM processes come from tt, DY, and multiboson production. Additionally, events from t⿱㇒t production in association with $\mathrm{Z}, \mathrm{W}$, or $\mathrm{H}$ bosons are significantly contributing in the signal regions. 
Well reconstructed $t \bar{t}$ events are contained in regions of low $M_{\mathrm{T} 2}(\ell \ell)$ and $M_{\mathrm{T} 2}(b \ell b \ell)$. Mismeasurements of $p_{\mathrm{T}}^{\text {miss }}$ or misidentification of leptons can promote events to regions with higher values of $M_{\mathrm{T} 2}(\ell \ell)$ and $M_{\mathrm{T} 2}(b \ell b \ell)$. Simulated events are used to predict the tit background in the signal regions. The modeling of these events is validated in dedicated control regions and their normalization is extracted by inverting the requirements on $M_{\mathrm{T} 2}(\ell \ell)$. A systematic uncertainty is assigned to the prediction based on the agreement of data and simulation in the control regions.
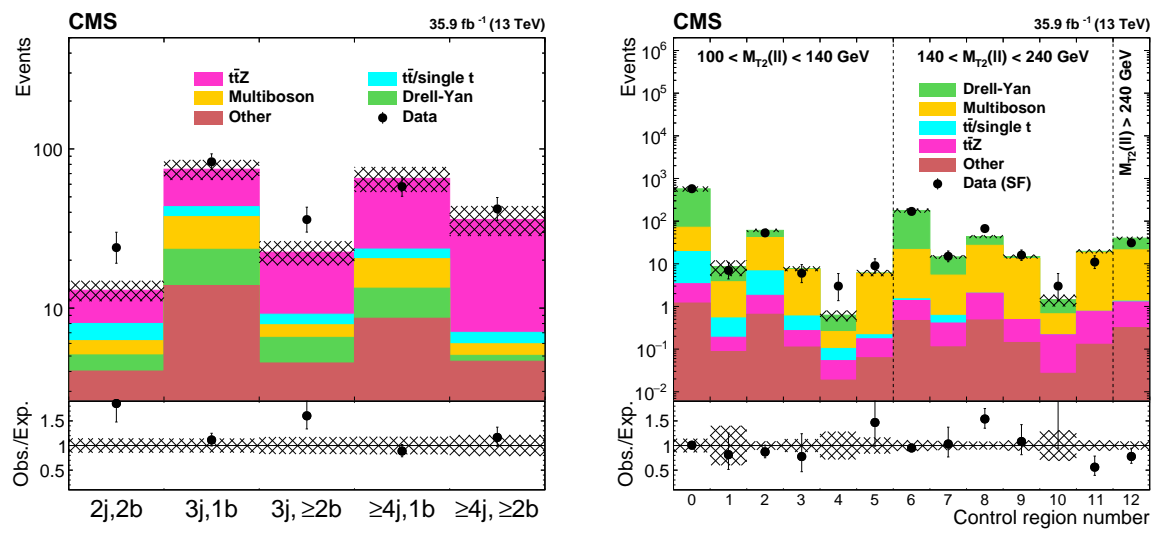

Figure 3: Event yields in the tṫ $Z$ (left) and Drell-Yan and multiboson control regions (right) with the relevant SM processes scaled to data via a maximum likelihood fit. The hatched band shows the uncertainties from the fit as well as uncertainties from experimental effects [1].

The overall normalization of events from $t \bar{t}$ production in association with a $\mathrm{Z}$ boson, which constitutes an irreducible background if the $\mathrm{Z}$ boson decays to neutrinos, is measured in final states with three leptons. We obtain sample of $\mathrm{t} \overline{\mathrm{t}} Z$ events with high purity by requiring at least one $\mathrm{b}$ jet and a same-flavor dilepton pair with an invariant mass close to the $\mathrm{Z}$ boson mass. Event yields in the dedicated control regions are shown in Fig. 3 (left). The measured data-tp-prediction scale factor which is applied to simulated distributions is compatible with unity.

We measure the data-to-prediction scale factor simultaneously for DY and multiboson production in events that do not contain a $b$ jet but a same-flavor lepton pair with an invariant mass that lies within $15 \mathrm{GeV}$ around the $\mathrm{Z}$ boson mass. The measured scale factors are close to unity for both DY and multiboson events, and the resulting event yields are shown in Fig. 3 (right).

\section{Results}

The observed $M_{\mathrm{T} 2}(\ell \ell)$ and $M_{\mathrm{T} 2}(b \ell b \ell)$ distributions are well described by the expected SM backgrounds as shown in Fig. 4. No significant excess over predicted SM background is seen in the signal regions, as shown in Fig. 5.

The results are interpreted in the context of several simplified SUSY and DM models. For the $\mathrm{T} 2 t t$ model results are combined with other CMS searches for top squark pair production in final states with a single lepton [4] and the all-hadronic final states [5]. In the combined result top squarks with masses of up to $1050 \mathrm{GeV}$ for a massless LSP are excluded at $95 \%$ confidence

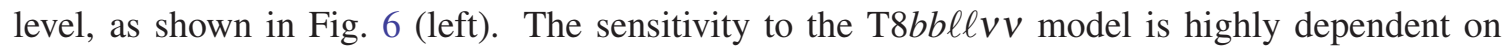
the masses of the intermediate chargino and slepton. In all considered configurations the chargino 

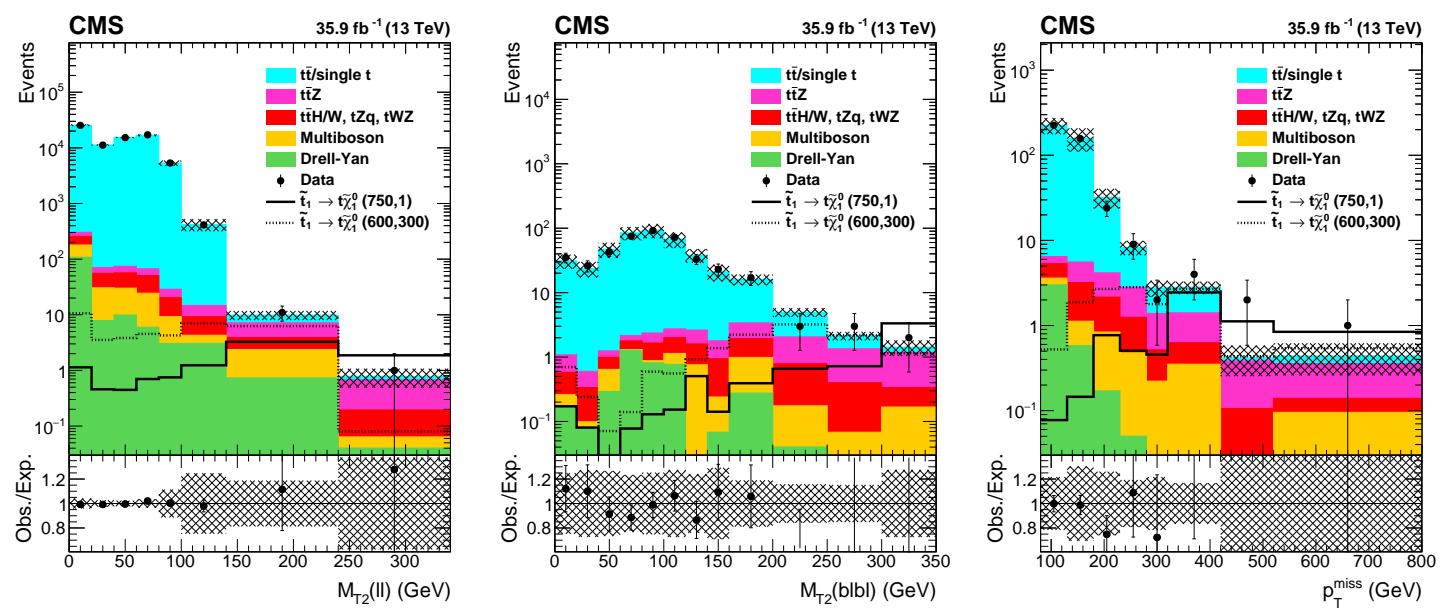

Figure 4: Distributions of $M_{\mathrm{T} 2}(\ell \ell)$ (left), $M_{\mathrm{T} 2}(b \ell b \ell)$ (center) and $p_{\mathrm{T}}^{\text {miss }}$ (right). For the $M_{\mathrm{T} 2}(b \ell b \ell)$ and $p_{\mathrm{T}}^{\text {miss }}$ distributions, events must satisfy $M_{\mathrm{T} 2}(\ell \ell)>100 \mathrm{GeV}$. The hatched band shows the total uncertainty on the expected SM background [1].

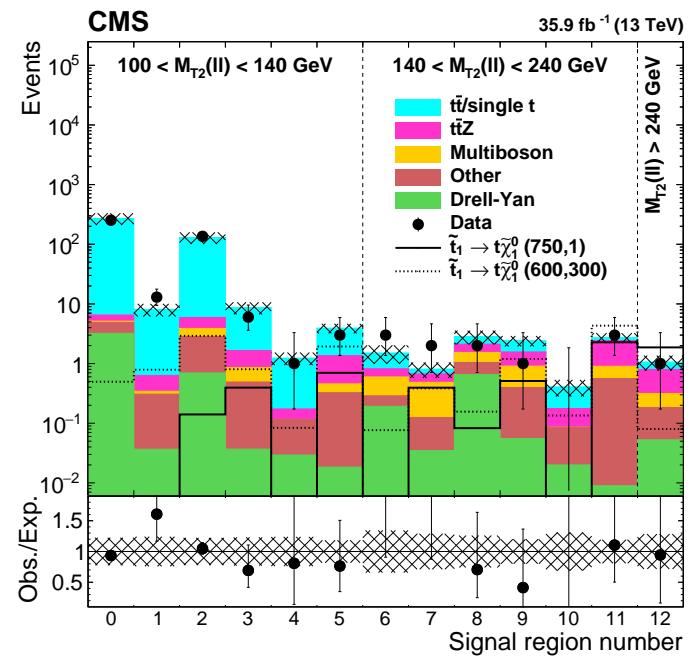

Figure 5: Predicted and observed event yields in the signal regions. The hatched band shows the total uncertainty on the expected SM background [1].

mass is set to the average of the top squark and the LSP mass. The slepton mass is parametrized by $m_{\tilde{\ell}}=x\left(m_{\tilde{t}_{1}}+m_{\widetilde{\chi}_{1}^{0}}\right)+m_{\widetilde{\chi}_{1}^{0}}$. If the masses of the slepton and the LSP are close, corresponding to low values of $x$, the sensitivity decreases due to the softer lepton $p_{\mathrm{T}}$ spectrum. For $x=0.95$, top squarks are excluded up to a mass of $1300 \mathrm{GeV}$ for an almost massless LSP, shown in Fig. 6 (right).

The simplified DM models assume DM particle pair production via a mediator that couples to top quarks and DM particles with a strength of $g_{q}=g_{\mathrm{DM}}=1$. Scalar and pseudoscalar mediators with masses of up to $100 \mathrm{GeV}$ and $50 \mathrm{GeV}$, respectively, are excluded for DM particle masses of $1 \mathrm{GeV}$, shown in Fig. 7.

In order to facilitate reinterpretation of the analysis, results in aggregate signal regions are provided, as well as correlation and covariance matrices for the full set of signal regions. The 

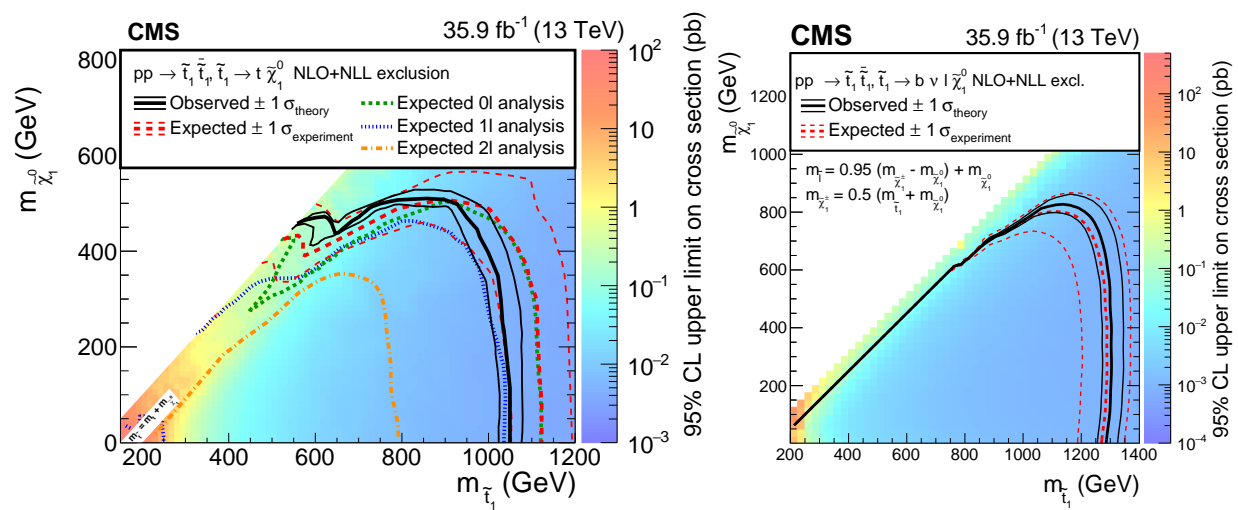

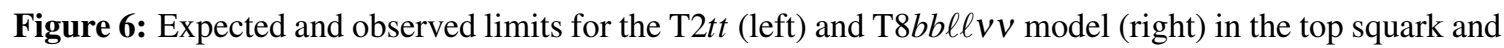
LSP mass plane. The color map indicates the 95\% CL upper limit on the product of cross section and the square of the branching fraction. The area below the thick black line represents the observed exclusion limit at $95 \% \mathrm{CL}$. The red dashed line corresponds to the expected limits of the combined result for the T2tt model

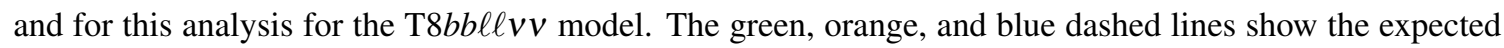
limits of the individual analyses (left) [1].

material can be accessed on HEPData [1], together with digitized versions of all figures and tables.
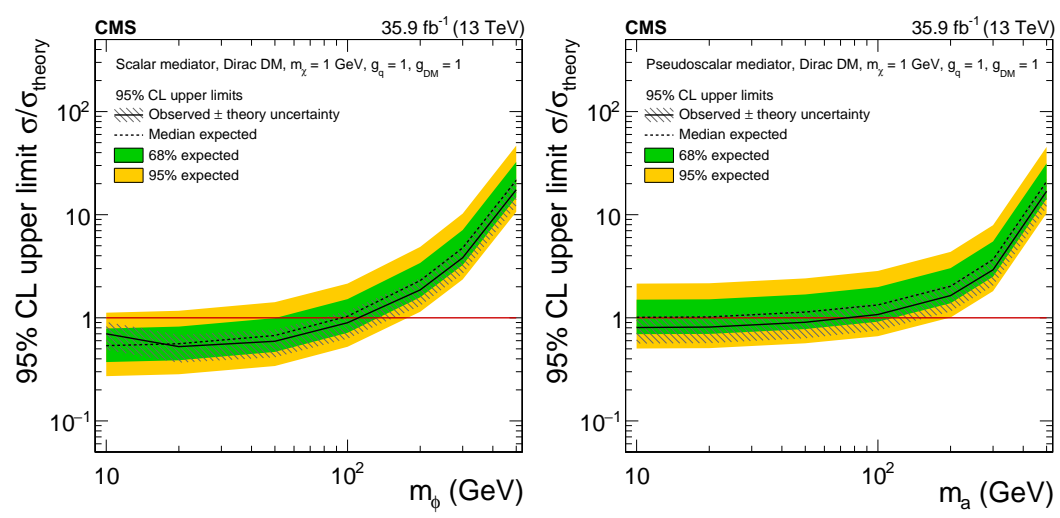

Figure 7: The 95\% CL expected (dashed) and observed limits (solid line) on $\mu=\sigma / \sigma_{\text {theory }}$ for a DM particle with $m_{\chi}=1 \mathrm{GeV}$. Different masses for the scalar (left) and pseudoscalar (right) mediators are assumed. The red horizontal line indicates $\mu=1$. The hashed area around the observed limit corresponds to the $30 \%$ theory uncertainty in the inclusive signal cross section [1].

\section{References}

[1] A. M. Sirunyan et al. [CMS Collaboration], Search for top squarks and dark matter particles in opposite-charge dilepton final states at $\sqrt{s}=13$ TeV, Phys.Rev. D97 (2018) 032009, [arXiv:1711.00752], [10.17182/hepdata.79809], [doi:10.1103/PhysRevD.97.032009].

[2] S. Chatrchyan et al. [CMS Collaboration], The CMS experiment at the CERN LHC, JINST 3 (2008) S08004, [doi:10.1088/1748-0221/3/08/S08004]. 
[3] S. Chatrchyan et al. [CMS Collaboration], Interpretation of searches for supersymmetry with simplified models, Phys.Rev. D88 (2013) 052017, [arXiv:1301.2175], [doi:10.1103/PhysRevD.88.052017].

[4] A. M. Sirunyan et al. [CMS Collaboration], Search for top squark pair production in pp collisions at $\sqrt{s}=13$ TeV using single lepton events, JHEP 10 (2017) 019, [arXiv:1706.04402], [doi:10.1007/JHEP10(2017)019].

[5] A. M. Sirunyan et al. [CMS Collaboration], Search for direct production of supersymmetric partners of the top quark in the all-jets final state in proton-proton collisions at $\sqrt{s}=13 \mathrm{TeV}$, JHEP 10 (2017) 005, [arXiv:1707.03316], [doi:10.1007/JHEP10(2017)005]. 